\section{Cahiers de littérature orale}

$67-68 \mid 2010$

Quand l'art prend la parole

\title{
Dialiba Konaté, ou celui qui donne à voir la parole des griots
}

\section{Cécile Leguy}

\section{OpenEdition}

1 Journals

\section{Édition électronique}

URL : https://journals.openedition.org/clo/488

DOI : $10.4000 /$ clo.488

ISSN : 2266-1816

Éditeur

INALCO

\section{Édition imprimée}

Date de publication : 15 janvier 2010

Pagination : 203-222

ISBN : 978-2-85831-196-5

ISSN : 0396-891X

\section{Référence électronique}

Cécile Leguy, « Dialiba Konaté, ou celui qui donne à voir la parole des griots », Cahiers de littérature orale [En ligne], 67-68 | 2010, mis en ligne le 20 septembre 2012, consulté le 30 juin 2021. URL : http:// journals.openedition.org/clo/488; DOI : https://doi.org/10.4000/clo.488

Ce document a été généré automatiquement le 30 juin 2021.

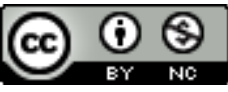

Cahiers de littérature orale est mis à disposition selon les termes de la Licence Creative Commons Attribution - Pas d'Utilisation Commerciale 4.0 International. 


\title{
Dialiba Konaté, ou celui qui donne à voir la parole des griots
}

\author{
Cécile Leguy
}

\section{NOTE DE L'AUTEUR}

Je remercie Dialiba Konaté pour sa collaboration et pour le temps qu'il a accepté de me consacrer, et Étienne Féau, conservateur en chef du Patrimoine (Musées de France), grâce à qui j'ai pu rencontrer Dialiba Konaté. Cet article a été rédigé suite à deux longs entretiens avec le dessinateur, le 26 octobre et le 6 novembre 2009.

Quand le griot ${ }^{1}$ vit le dessin que Dialiba Konaté avait fait de Soundiata, il s'exclama: " c'est la première fois que je vois dans mes yeux ce que j'ai depuis toujours dans la tête et dans les oreilles!»

(Féau, 2000 : 31)

Blotti contre sa mère, l'enfant découvre avec elle, en cheminant de dessin en dessin, le récit légendaire du héros fondateur d'un empire dont le nom résonne à ses oreilles comme un appel. Toute une histoire - qu'on ne lui a jamais racontée comme il ne manquera pas de le reprocher à ses parents ensuite - se déploie sous ses yeux tant étonnés qu'émerveillés. Des hommes aux muscles saillants, drapés de cotonnade brune, le chef orné de cauris, se présentent à lui dans toute leur majesté, armés de lances et de flèches, victorieux. Dans un album adressé aux enfants, l'histoire du Mali se raconte à travers les dessins expressifs de Dialiba Konaté.

2 Les héritiers de l'empire mandingue n'ont pas pour habitude de matérialiser la parole, ni par l'écriture ni par d'autres moyens plastiques ${ }^{2}$. Le récit des épopées ${ }^{3} \mathrm{y}$ est réservé à certains griots qui suivent pour cela une formation spécifique. Le travail de Dialiba Konaté est particulièrement original, car il utilise le dessin pour transmettre les paroles des griots, donnant un support inédit aux légendes ancestrales de l'Afrique de l'Ouest. En effet, si l'épopée de Soundiata, sujet du premier album publié par Dialiba Konaté 
(2002), a déjà fait l'objet de différentes publications écrites depuis les années 1960 (Niane, 1960 ; Diabaté, 1975 ; Innes, 1974 ; Johnson, 1980 ; Cissé et Kamissoko, 1988 et Jansen, 1995 pour les versions les plus connues), ces travaux sont le fruit de collectes et de traductions effectuées par des chercheurs dans le cadre d'une démarche scientifique. Une telle présentation fondée sur le dessin est totalement inédite, dans un contexte où le dessin n'est pas un art couramment pratiqué.

Empreints de réalisme, riches de détails et de précision, les dessins de Dialiba Konaté font cependant la part belle à l'imaginaire. Ils servent des récits hauts en couleur, adaptés pour un public de jeunes lecteurs par l'éditeur. Dialiba Konaté lui-même accompagne généralement ses croquis de textes explicatifs, sur le mode des bandes dessinées d'antan, l'image donnant à voir ce que le texte évoque en quelques mots. Faire parler le dessin pour transmettre à travers son art les paroles entendues autrefois de la bouche des anciens et des griots, telle est l'ambition de Dialiba Konaté, artiste mais surtout passeur, passionné d'histoire comme il aime à se définir lui-même.

\section{Un don précoce pour le dessin}

4 Né au Sénégal en 1942, Dialiba Konaté a passé une partie de son enfance à Bandiagara, à l'est de l'actuel Mali, auprès de son tuteur Ben Omar Sy, enseignant qui l'a initié à l'histoire, et de son grand-père maternel, Demba Sadio Diallo. Ce grand-père, ancien fonctionnaire ${ }^{4}$ apprécié d'une grande érudition, était un ami intime d'Amadou Hampâté Bâ $\hat{a}^{5}$ qui venait régulièrement le consulter ${ }^{6}$. Demba Sadio Diallo aimait parler d'histoire, de politique, de religion. Les anciens de Bandiagara discutaient chez lui, de longues heures durant, et le jeune Dialiba les écoutait sans rien dire, aimant seulement être présent. C'est ce grand-père, érudit et sage, qu'il présente comme son modèle dans sa quête passionnée de connaissance et de compréhension. Suivant ses traces en effet, il retourne à l'écoute des anciens et des griots, lors des séjours qu'il fait chaque année au Mali auprès de sa troisième épouse. Comme lui également, il aime multiplier les sources de savoir et passe de longues heures à la bibliothèque du centre Beaubourg, à lire les travaux des ethnologues ou les textes retranscrits de la tradition orale. Car c'est d'abord comme historien qu'il présente sa mission: son travail est fondé sur une documentation précise, une attention aux variations, un souci du détail et de la précision. Sa passion pour l'histoire ne se réduit d'ailleurs pas aux anciens empires qui ont marqué l'Afrique de l'Ouest : les événements plus récents l'inspirent également et, en parcourant les piles de dessins qui jonchent le canapé du salon familial qui lui sert de bureau à Paris, on reconnaît les portraits accompagnés de commentaires de Modibo Keïta, d'Alpha Oumar Konaré ou d'ATT, de Nasser ou bien encore de Senghor, chacun emballé précautionneusement dans une feuille de plastique. 


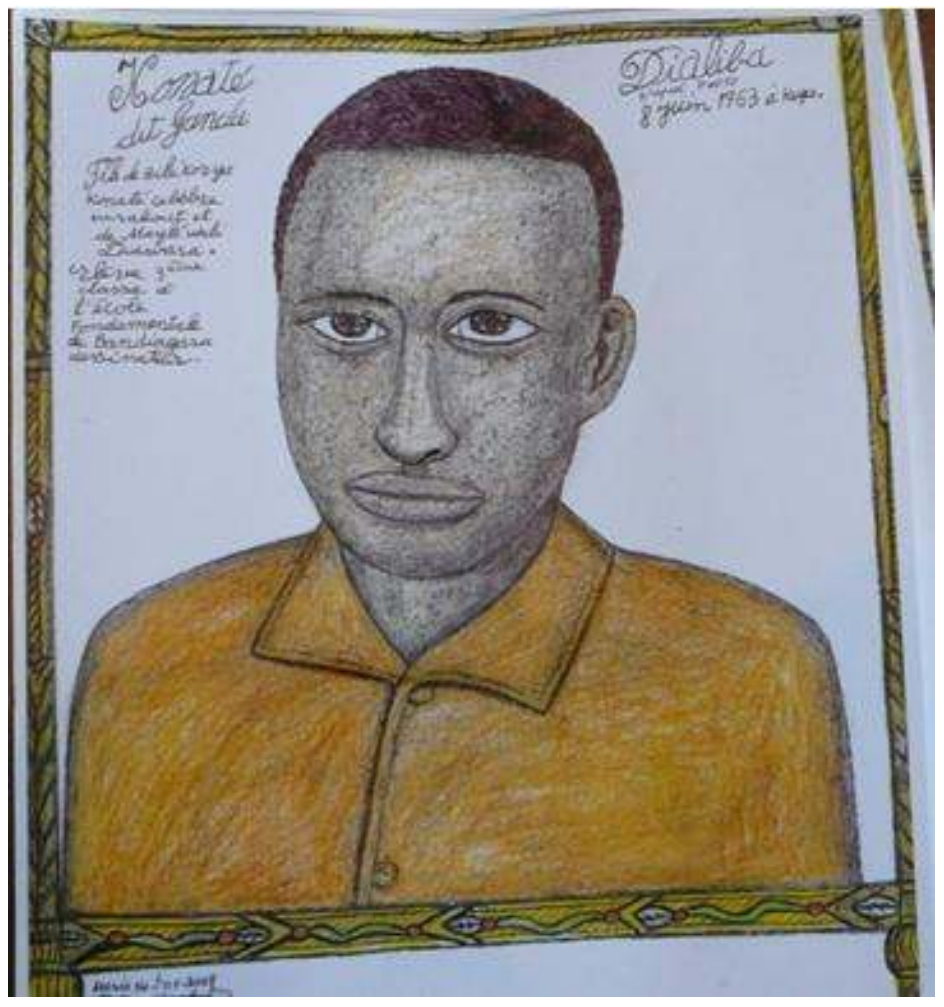

Dès ses premières années d'école, Dialiba s'était fait remarquer pour ce qu'il considère comme un "don». Le maitre lui permettait de dessiner au tableau les cartes de géographie et les croquis nécessaires aux leçons de sciences. On connaissait son aptitude à reproduire avec précision ce qu'il observait. Mais il aimait aussi les histoires : il raconte ainsi le plaisir qu'il avait, enfant, à mettre en scène les silhouettes d'un théâtre d'ombres derrière une bande de coton blanche éclairée par une bougie. Ses enseignants s'intéressaient à ses dessins et, apprenant que les prêtres de la mission catholique organisaient un concours d'art, ils lui proposèrent d'y participer. Il s'agissait de dessiner la statue de Socrate. Lauréat du concours, il fut alors invité à partir pour deux ans dans une école des Beaux-Arts en Union soviétique. Il avait dix-neuf ans, sa mère refusa qu'il parte si loin, dans un pays où ne résidait aucun parent. Mais cette réussite avait conforté sa passion pour le dessin et quand il arriva à Paris quelques années plus tard, en 1965, il chercha immédiatement à suivre des cours. Se souvenant du concours de dessin organisé par les missionnaires, il entra dans une église et demanda conseil au prêtre. Comme il travaillait toute la journée, celui-ci lui indiqua des cours du soir, dans une école d'art de la ville à Montmartre d'abord (école dont il garde précieusement la carte d'inscription pour l'année 1965-66 dans son portefeuille), puis aux Arts déco dans le quartier de l'actuel Beaubourg. Ses horaires de travail ne lui donnaient guère de disponibilité et il s'inscrivit ensuite aux cours de l'école $A B C$ de Paris, cours par correspondance dispensés par des enseignants passionnés qui n'hésitaient pas à lui rendre visite à domicile pour lui prodiguer des conseils et évaluer ses travaux. Dialiba Konaté a conservé de ce temps les exercices de drapés, de nus et de paysages, annotés par ses professeurs, en particulier Georges Pacouil (1903-1997) qui savait l'encourager tout en laissant libre cours à son talent propre. 
Le dessinateur eut ensuite la possibilité d'intégrer l'Université Paris-VIII à Vincennes et d'y suivre une formation complète en Arts plastiques, validant les unités de valeur du DEUG jusqu'au DEA, en suivant des cours le soir et le samedi. En 1977, Paris-VIII était encore un centre universitaire expérimental ouvert à la diversité, accueillant des étudiants non bacheliers, qui proposait des enseignements originaux qu'on ne connaissait pas dans les cursus universitaires académiques. Dialiba Konaté valide des U.V. dont les intitulés sonnent comme des programmes enchanteurs: «Atelier de communication visuelle", "Pratique de la bande dessinée», "Manifestations artistiques, expositions, musées d'art ", "Introduction à la sémiologie des arts plastiques ", "Bande dessinée et roman-photo", "Études cinématographiques et audiovisuelles sur le thème "Cinéma et tiers-monde" "... Il s'intéresse tout particulièrement aux cours de théorie et d'histoire de la bande dessinée animés par Jean-Louis Chazelas, et acquiert ainsi tout un savoir-faire qu'il va mettre en pratique pour transcrire sur papier cartonné les histoires du passé qui résonnent dans sa tête.

\section{Coné, célèbre guérisseur de Kolokani (1994)}

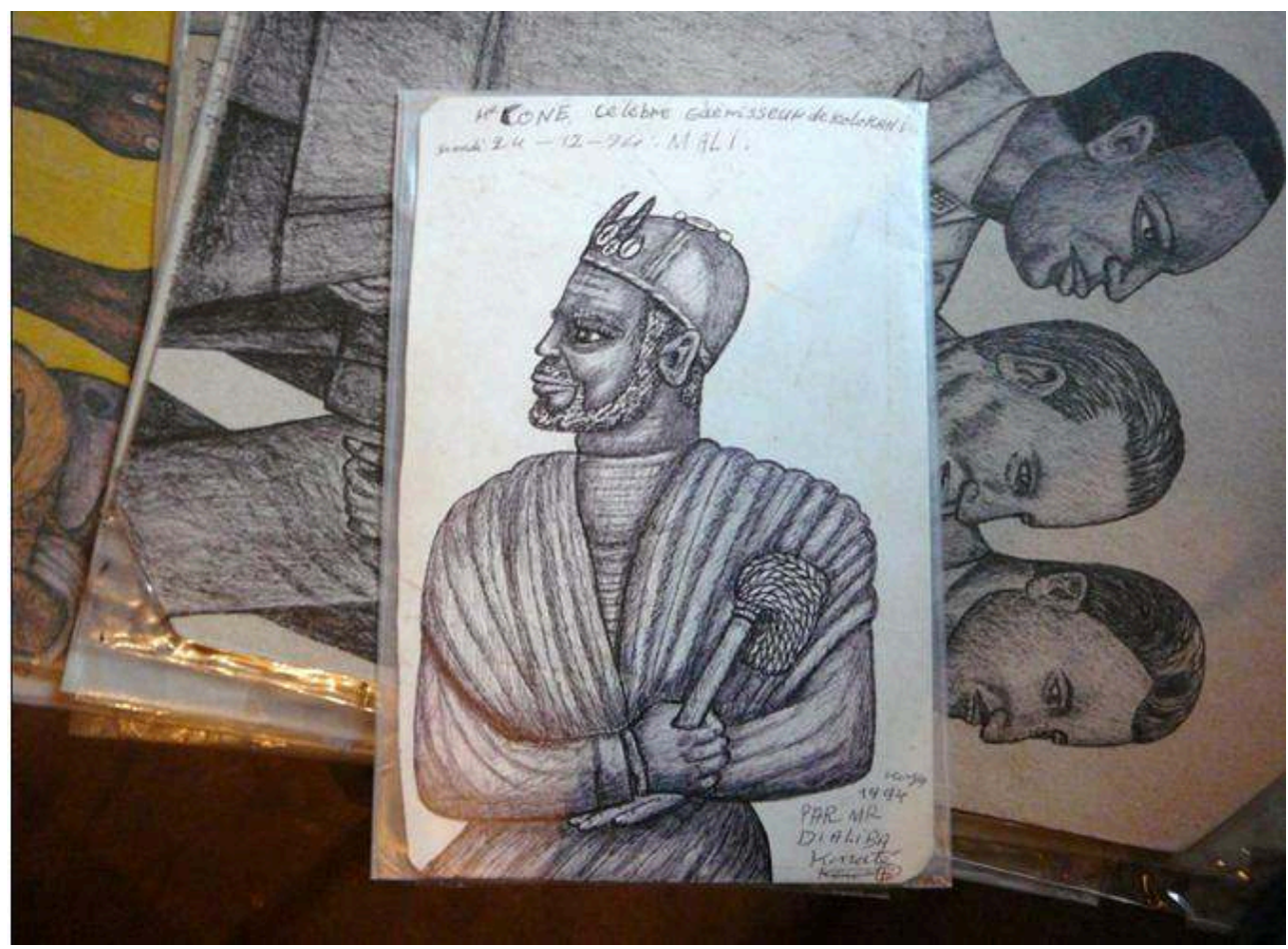

(Au second plan, on reconnaît Tito, Nasser et Modibo Keïta à la conférence de Belgrade de 1961).

7 Pour élaborer ses dessins, Dialiba Konaté utilise une technique qui lui est tout à fait personnelle. La plupart sont réalisés sur des feuilles cartonnées de format A3, qu'il présente généralement à l'italienne. Le dessin remplit la feuille mais quelques commentaires écrits au stylo à bille le complètent dans un coin. Le dessin lui-même est entièrement fait au stylo à bille, avec lequel sont travaillés volumes et modelés. Ensuite, c'est au crayon de couleur que Dialiba Konaté donne la touche colorée à l'ensemble. Le dessin terminé est passé à la laque pour cheveux, produit qui tout en fixant les couleurs, les étale quelque peu en leur donnant un aspect brillant. Cette méthode, que l'on peut qualifier d'artisanale, donne à l'ensemble un aspect qui peut être perçu comme « naï », mais dont la profondeur est cependant éloquente. 
8 C'est d'abord dans le cadre de son travail chez Fina que Dialiba Konaté eut l'occasion d'exposer ses premiers dessins, sur la proposition du comité d'entreprise qui organisait des concours. Les dessins, qui avaient plu à ses collègues, furent ensuite présentés dans différents lieux comme l'hôtel Concorde ou Radio France. Une présentatrice de la troisième chaîne de télévision française lui proposa de les faire découvrir aux téléspectateurs dans son émission du matin, "Mosaïque ». C'était en 1982. Plusieurs personnes commencèrent alors à s'intéresser à son travail. Refusant, sur le conseil de Jean-Louis Chazelas, son professeur de bande dessinée, de vendre ses planches de peur qu'elles ne soient dispersées, Dialiba Konaté choisit de les confier au Musée National des Arts d'Afrique et d'Océanie (MAAO) - sur la proposition de Colette Noll, alors conservateur des Arts africains au MAAO, qui avait vu l'émission de FR3 et avait demandé à le rencontrer - car il craint qu'en les conservant chez lui, elles ne soient abîmées par les enfants. Celui qui se perçoit lui-même comme un simple passeur de paroles anciennes envisage alors que la valeur de son travail puisse dépasser celle qu'il y accorde lui-même. Mais son objectif n'est pas de « faire de l'art » et il se défend d'être un artiste, qualificatif qui ne lui plaît guère du fait de sa connotation dilettante. Dialiba Konaté se conçoit comme un historien, et dès le départ il tient - et il y tient toujours autant aujourd'hui - à transmettre d'abord l'histoire de l'Afrique aux jeunes générations qui n'ont pas eu comme lui l'occasion d'entendre les griots et les anciens discuter chaque jour. Son ambition est avant tout de transmettre, avec le plus de justesse possible, les valeurs du passé aux plus jeunes.

9 Le rêve a trouvé les moyens de se réaliser à la fin des années 1990, d'une part grâce à une série d'expositions (dont dès 1983 une participation au Salon de la bande dessinée d'Angoulême ${ }^{7}$ ) et d'interventions dans des collèges et des médiathèques, puis par le travail éditorial. L'événement le plus important qui eut un réel impact sur la carrière du dessinateur fut sans nul doute l'exposition qui eut lieu au Musée National des Arts d'Afrique et d'Océanie (MAAO) de la Porte Dorée en 2000-20018. Grâce à Étienne Féau, qui était à cette époque en charge des collections d'Art africain au MAAO, deux cents dessins ont été présentés au public trois mois durant. Chaque samedi après-midi, des griots venaient animer le musée en clamant l'épopée de Soundiata. L'exposition reçut un franc succès'. C'est à cette occasion que la production de Dialiba Konaté a retenu l'attention de Pierre Koernig, lui-même auteur de bandes dessinées, qui s'est passionné pour ce travail et a établi le premier contact avec Brigitte Morel des éditions du Seuil, envisageant une publication de l'épopée de Soundiata sous la forme d'un album adressé aux enfants. Un important travail éditorial fut alors fait pour retenir cinquante planches parmi les deux cents qui étaient exposées. L'écrivain Martine Laffon reprit le texte accompagnant chaque dessin pour en proposer une adaptation littéraire plus accessible au lecteur. L'album publié en 2002 fascina les professionnels du livre pour enfants par son originalité, sa beauté, sa taille hors norme rendant justice aux dessins, et surtout pour sa fidélité au contexte africain si souvent malmené dans les publications adressées à la jeunesse (Godin, 2005). Après l'exposition du MAAO, le succès de ce premier album contribua à encourager Dialiba Konaté à mettre son talent au service de l'histoire de l'Afrique de l'Ouest ${ }^{10}$. Lors de nos entretiens à l'automne 2009, il terminait les dernières fresques de l'histoire du Wagadu, l'empire du Ghana qui a précédé dans le temps l'empire du Mali de Soundiata (Konaté, 2010). 
Le canapé familial, préparation du futur « Empire du Ghana » (octobre 2009)

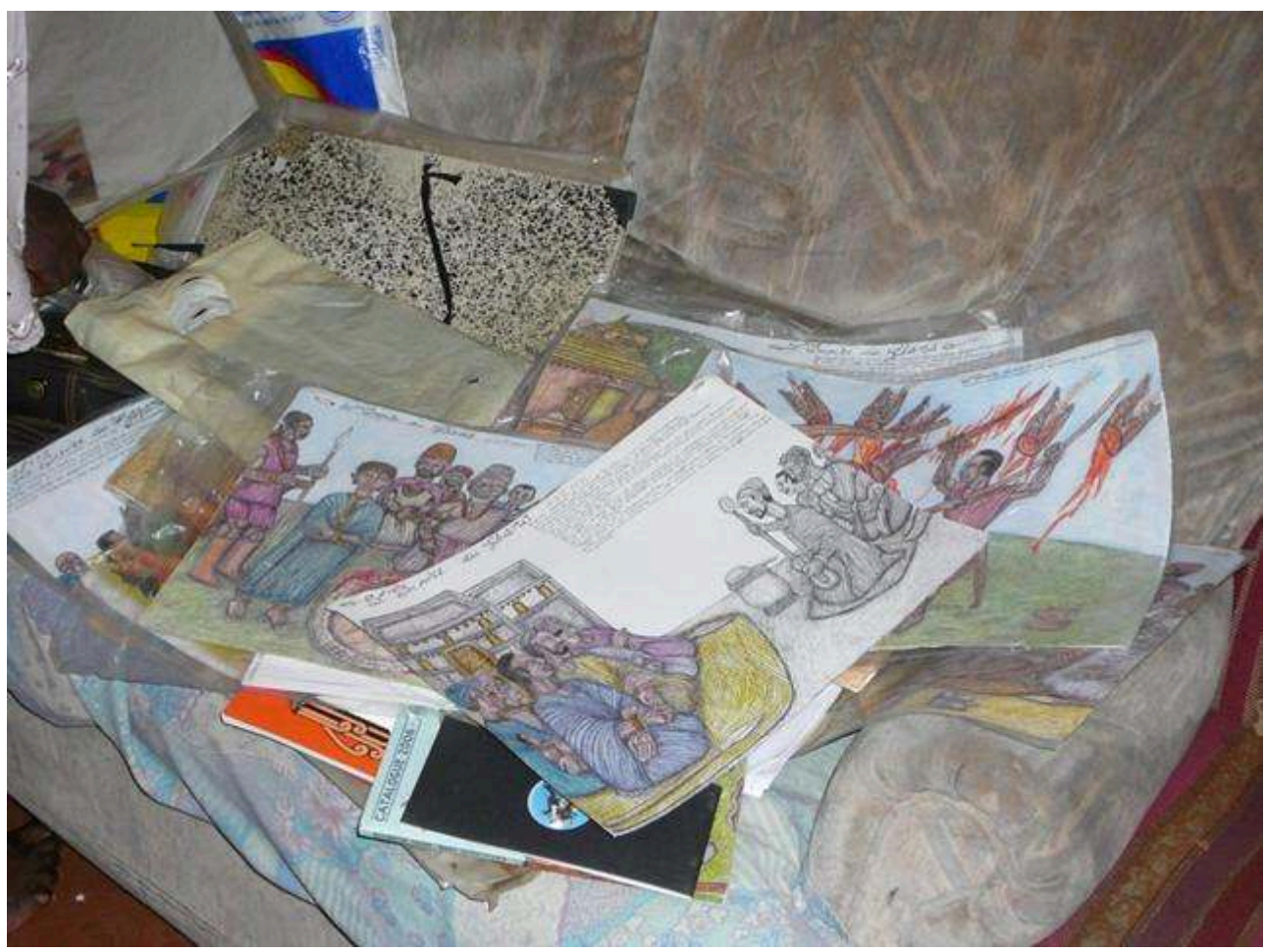

\section{Deux rencontres fondatrices}

S'il ne se conçoit pas comme artiste, mais comme quelqu'un qui a reçu un don et s'est donné pour mission d'en user pour transmettre, sous une forme inédite, les paroles et savoirs ancestraux de sa région d'origine, Dialiba Konaté, en faisant le récit de son parcours, présente deux rencontres extraordinaires qu'il considère comme des événements déclencheurs à l'origine de sa production.

11 En 1975, Dialiba Konaté rencontre au Sénégal un grand griot qui avait étudié à Kangaba $^{11}$. Il l'écoute raconter l'épopée de Soundiata et apprécie sa manière imagée de dire l'histoire. Sa parole est tellement évocatrice qu'il rend visibles les événements qu'il relate, non seulement par la description des détails, mais aussi par le rythme et l'agencement des mots qu'il utilise. Ainsi, en racontant comment le jeune infirme, fils de Sogolon la bossue, réussit à se redresser et à devenir le héros que l'on sait, il énonce en montant progressivement la voix : "Soundiata grosse tête; Soundiata gros yeux; Soundiata le nez épaté ; Soundiata, lèvres émincées; Soundiata gros cou, gros bras ; Soundiata taille élancée; Soundiata gros pied; Soundiata le généreux... ». Ce véritable chant à la gloire du héros mandingue touche Dialiba Konaté qui se met alors à le dessiner comme il le voit, comme le récit du griot le fait voir : yeux, nez, muscles... Montrant son dessin au griot, celui-ci est subjugué de reconnaître son personnage, celui qu'il a devant les yeux quand il raconte l'épopée. Il s'exclame alors: "Ça c'est Soundiata !»Ce dessin, fait comme sous la dictée en écoutant le récit du griot, a joué un rôle déclencheur important dans la démarche de Dialiba Konaté. La décision de transmettre par le dessin les récits des anciens que lui-même avait reçus par la parole était prise. Dialiba Konaté n'est pas griot, mais il sait communiquer par le dessin. Il lui semble d'autant plus important de mettre ce don au service de la transmission des 
traditions orales qu'il vit en France et y voit grandir ses enfants, loin des sources de savoir auxquelles il a puisé lui-même. Ses enfants sont scolarisés, ils regardent la télévision, arpentent leur quartier parisien et connaissent peu la terre africaine de leurs parents. Mais ils s'intéressent aux dessins de leur père, demandent à connaître les aventures des héros dont ils voient les portraits. Pour les enfants aussi, les siens et tous ceux des immigrés qui grandissent loin des lieux de parole des anciens, il estime alors qu'il est de son devoir de mettre en scène, dans une suite de dessins éloquents, ce que lui-même a entendu raconter dans son enfance.

12 Méticuleux, Dialiba Konaté cherche la précision pour que ses dessins soient les plus fidèles possible à la réalité historique. Pour mener à bien son entreprise, il a besoin de détails qu'il ne trouve pas dans les travaux des chercheurs qu'il consulte à la bibliothèque. C'est alors qu'il croise la route d'un autre personnage extraordinaire, qu'il nomme «l'initié ». C'est un personnage imposant, vêtu d'une tenue traditionnelle en bogolan sur laquelle sont cousus des cauris et autres talismans, qu'il rencontre un jour dans le train de Dakar. Corpulent, majestueux, il porte une coiffure munie de cornes qui effraient les musulmans comme les chrétiens. Les gens le craignent et évitent de s'asseoir trop près de lui, par méfiance envers les pouvoirs occultes qu'ils lui imaginent. Fasciné par le personnage, Dialiba Konaté s'approche et demande à lui parler, ce que l'autre accepte sans hésiter. Il se présente comme un grand guérisseur, parti à Dakar à la demande de quelqu'un dont le fils est épileptique, afin qu'il use de sa science pour le soigner. Ce guérisseur, bien qu'assis dans le train et muni d'une radio, semble venir de temps immémoriaux. Dialiba Konaté lui explique son désir de dessiner l'épopée de Soundiata et lui demande s'il peut lui décrire la manière dont étaient faits vêtements et parures au XIIIe siècle. Après quinze minutes de silence, pendant lesquelles le dessinateur pense qu'il refuse de répondre, il prend la parole.

Il a dit Banfoula, une sorte de coiffure à deux battants qui ressemble un peu aux dents du caïman, ils faisaient comme cela, des fois ils mettaient des cauris, des fois sans cauris. Ils portaient aussi des calottes, une sorte de calotte comme ça, mais deux battants. Ça on portait pour aller faire la guerre ou pour aller à la chasse. Ils portaient aussi des habits amples, mais sans poche souvent. Souvent ils mettaient des poches. Et puis des pantalons un peu courts, ça descend jusqu'après le genou quoi, ou bien ça s'arrête au genou. C'est les habits amples, au-dessus. Mais quand ils allaient aux champs, ou bien à la chasse ou à la guerre, ils portaient des habits courts, des pantalons courts et puis des blouses courtes attachées avec une ceinture, des tuniques. Et puis ils portaient des sandales ; des sandales il m'a montré comment on fait. Après il dit: les femmes tressaient leurs cheveux, souvent sans foulard: le foulard n'était pas obligatoire à l'époque. Elles portaient des habits amples, sans poche, un peu comme les hommes. Les habits étaient un peu comme ceux des hommes, un peu différents. Bien juste quoi. Cela leur allait bien. Après qu'il m'ait parlé, j'ai repensé à tout ce qu'il m'avait dit et j'ai travaillé là-dessus. J'ai commencé mes dessins. C'est comme cela que j'ai commencé à dessiner Soundiata.

(Entretien avec Dialiba Konaté, 26 octobre 2009) 
Soundiata Keïta en habit de chasseur (1984)

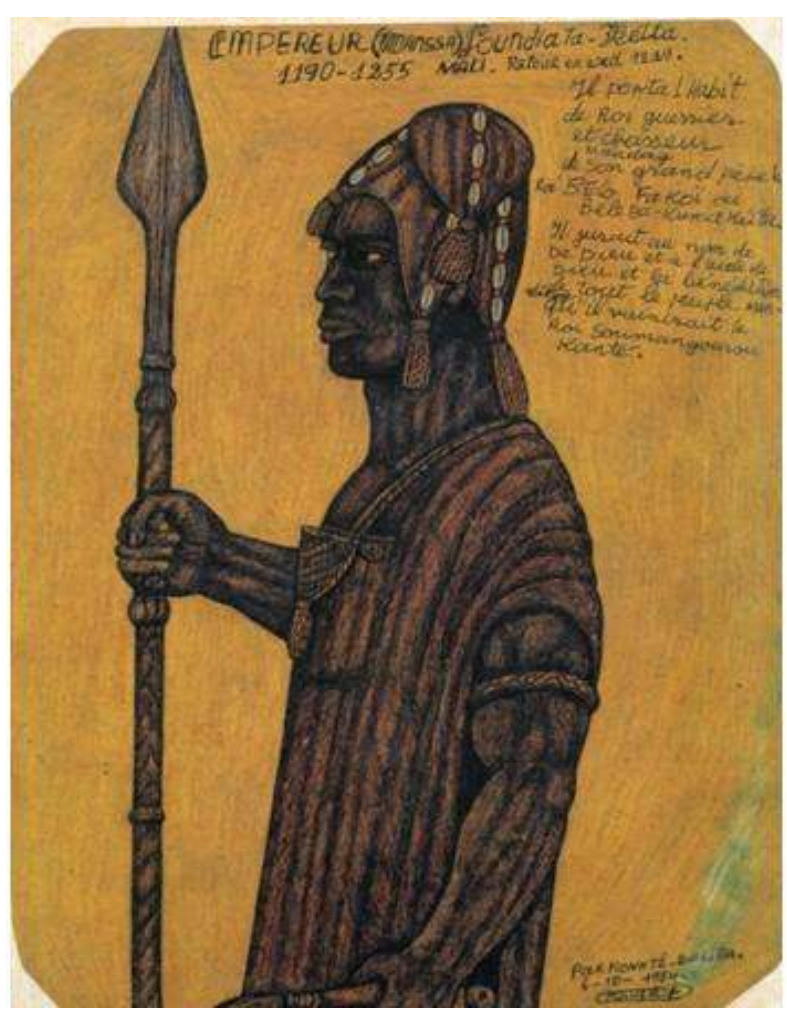

(dessin publié dans Konaté, 2002)

13 La réponse de "l'initié » va lui permettre de parfaire ses dessins, de leur donner les détails qui manquaient et, surtout, de dépasser la seule dimension historique pour atteindre une dimension plus mystique qu'il cherche également à transmettre. Car les histoires anciennes ne sont pas pour lui seulement des récits du passé, mais d'abord des leçons de vie qui gardent toute leur actualité.

\section{Peut-on mettre la parole en image?}

Si Dialiba Konaté avait déjà pris la décision de dessiner pour transmettre l'histoire de ses ancêtres à ses enfants, un autre élément va l'entraîner vers la forme du récit en dessins. À l'Université de Vincennes, qu'il va fréquenter à partir de 1977, on demande, pour valider un enseignement de bande dessinée, de raconter un épisode historique, en relation avec le pays d'origine de chaque étudiant. Ce sujet pour un travail universitaire va devenir le support d'une vie de dessinateur. Dialiba Konaté se remémore alors le récit maintes fois entendu de la fondation de l'empire du Mali, se procure quelques ouvrages spécialisés et commence à concevoir les différents dessins de sa bande dessinée. Chaque dessin est fait sur une feuille de carton, récupérée parfois à l'occasion de l'achat d'une chemise. Le moindre croquis fait l'objet d'une recherche pour correspondre le plus précisément possible à l'épisode relaté, pour n'omettre aucun détail. Ainsi, Dialiba Konaté part d'abord de la parole, celle qu'il a entendue enfant auprès des griots et de son grand-père, celle qu'il continue de solliciter lors de ses séjours au Mali. Il entreprend ensuite des recherches dans les livres, auprès des travaux spécialisés, afin de faire des recoupements. S'il veut s'assurer d'un détail, il demande à sept ou huit personnes différentes et qui ne se connaissent pas de lui donner des 
précisions. Quand les renseignements concordent entre ses différentes sources orales, quand les écrits des ethnologues confortent ses informations, il peut alors envisager le dessin dans ses moindres détails. On peut ainsi le rencontrer, installé à la bibliothèque du Centre Beaubourg, occupé à fignoler un croquis après avoir eu confirmation du déroulement d'un événement à la lecture d'un chapitre de Monteil, de Delafosse ou encore d'Ibn Khaldoun.

Le mémoire demandé par l'université a reçu comme titre « Histoire, géographie, art et traditions du Manding ", et fut le noyau initial d'une grande série de dessins, qui se sont petit à petit amoncelés au domicile de leur auteur. Ce ne sont pas seulement les récits épiques ou les personnages historiques qui l'inspirent, mais aussi les traditions ancestrales, de manière générale, comme le montre bien l'album titré Les saisons oubliées : une enfance africaine (2007), choix de fresques retraçant les différents moments de la vie et prétexte à évoquer les souvenirs d'une "enfance africaine »: naissance, initiation, mariage, fête villageoise, marché, travaux des champs... mais rappelant aussi la vie de certains héros célèbres dont les griots honorent le nom dans leurs récits. Grâce à son don pour le dessin, toute parole peut donc ainsi être présentée, sous la forme de dessins colorés et évocateurs. Ayant souvent entendu dans son enfance les marabouts relater des récits bibliques, Dialiba Konaté souhaite également transcrire avec ses crayons les histoires imagées d'Adam et Ève ou de l'Arche de Noé (Konaté, 2005). C'est alors qu'une question vient le tarauder. Tant qu'il consacre ses dessins aux histoires anciennes ou aux portraits des hommes politiques du monde moderne, il ne s'interroge pas sur la légitimité de sa production d'images. Souhaitant aborder les représentations religieuses, il est confronté à l'aniconisme prôné par la tradition islamique. Si on ne trouve pas de représentation humaine dans les mosquées, si le Coran n'est généralement pas illustré12 et si de nombreux hadiths condamnent toute représentation humaine ${ }^{13}$, est-il légitime d'entreprendre une mise en image de la parole biblique? Pieux musulman, fils de marabout et souhaitant ne pas commettre d'impair, Dialiba Konaté décide alors de consulter un imam avant de commencer tout dessin sur ce sujet. L'imam lui répond sagement et l'encourage dans son entreprise, l'invitant à se référer au Coran et à ignorer les hadiths, pour lesquels les hommes se disputent alors qu'ils ont été écrits bien après la mort du prophète. Dans le Coran, la seule mise en garde énoncée concerne en effet les idoles, comme dans les autres traditions monothéistes. L'imam rassure donc Dialiba Konaté en lui précisant que tant que le dessin est plat, il n'y a pas de problème. Une représentation sous forme de statue serait plus problématique, dans la mesure où une statue reprend les formes humaines, reproduisant ainsi l'acte de création. Mais le dessin, mise à plat de la réalité, ne présente aucun risque. Réconforté par ces paroles, Dialiba Konaté a pu sans crainte retracer les principaux épisodes du « Récit des origines » et continuer avec confiance à mettre sur papier toutes les histoires entendues au cours de sa vie. 
Dialiba Konaté présentant un dessin en chantier pour son histoire de l'empire du Ghana (octobre 2009)

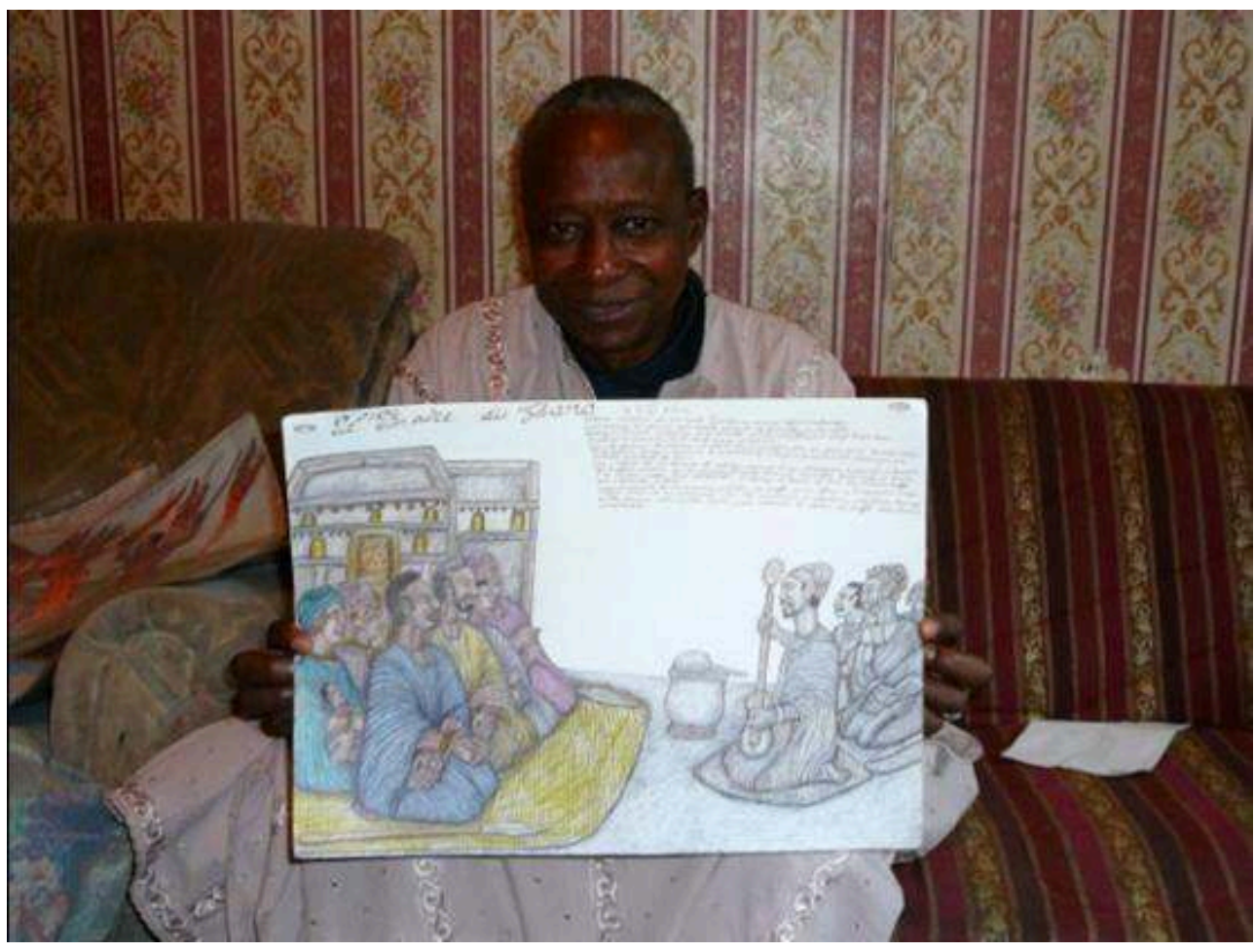

17 Quant aux griots, "gens de la parole " comme les nomme Sory Camara (1992), ils semblent apprécier cette mise à plat des récits épiques du passé. Selon Dialiba Konaté, «Les griots sont très contents quand ils voient cela. Ils disent que comme ça, on ne dira pas qu'ils parlent dans le vide.» (entretien avec Dialiba Konaté, 26 octobre 2009) La mise en image n'est pas incompatible avec l'art des griots et sans doute estiment-ils, dans une société où celle-ci prend de plus en plus de place, qu'elle peut être un support bienvenu pour les récits transmis de génération en génération par la seule oralité.

\section{Le choix des couleurs}

Les visiteurs des expositions avaient été subjugués par l'éclat des couleurs des dessins de Dialiba Konaté, que rendent assez bien les éditeurs dans les albums proposés pour la jeunesse. Chaque croquis a en effet été patiemment colorié, à l'aide de crayons de couleur comme ceux qu'on offre aux enfants. Interrogé sur le choix des couleurs, Dialiba Konaté n'évoque dans un premier temps que la «triade fondamentale » - blanc, rouge, noir - dont Turner avait montré le rôle primordial dans les conceptions magicoreligieuses, à partir de son ethnographie ndembu et de nombreuses comparaisons (Turner, 1972).

Les couleurs sont du langage. Il y a trois couleurs symboliques en Afrique: la couleur blanche, la couleur rouge, la couleur noire. La couleur rouge, ça symbolise la protection, contre les mauvais sorts, contre les mauvaises choses, c'est pourquoi un nouveau marié, une nouvelle mariée, on mettait quelque chose autour de son cou, rouge, pour la protection; la couleur noire, c'est que vous soyez invisibles devant les ennemis, c'est aussi pour la protection. Mais d'un autre côté, c'est tout ce qui est maléfique; et puis la couleur blanche, c'est que tout ce qui est caché, soit 
dévoilé. La vérité. Donc vous voyez, les couleurs aussi parlent.

(Entretien avec Dialiba Konaté, 6 novembre 2009) boîte. Il habille ses personnages de drapés de teintes différentes, certains en vert, d'autres en violet, en bleu ou en orange. Le fond du dessin lui-même est fait d'une sorte de lavis au crayon dont la couleur est choisie en fonction de l'ambiance. Relancé sur cette profusion de couleurs, il reprend alors une théorie de leur symbolisme qu'il semble réciter et dont on peine à déterminer l'origine. Selon cette théorie, le vert est «putréfaction, résurrection, fécondité, croyance » et le rouge "la couleur sang: vie, passion, l'amour divin, et sacrifice et symbole de l'enfer. C'est aussi le symbole de la terre ». La vie est « tout ce qui est putréfiable », précise Dialiba Konaté, c'est pourquoi le vert est « une couleur très intéressante pour un milieu de croyants ». Les tiges du mil et les feuilles du karité, mais aussi le vêtement de certains personnages, sont coloriés de vert. Le dessinateur aime aussi utiliser le violet, symbole d'une puissance passagère, d'un pouvoir éphémère et du "secret trouble ». C'est une teinte qui renvoie au passé et qui peut s'appliquer au vêtement d'un personnage au pouvoir temporel relatif tout comme au milieu général, pour retranscrire l'aspect trouble d'une ambiance, quand un secret pesant et néfaste semble écraser le ciel. À l'inverse, il aime utiliser le jaune, comme symbole de la lumière, de la clarté. Le jaune et le bleu clair sont souvent sollicités pour des scènes qui se passent dans la clarté du jour. Le jaune renvoie également à l'intelligence. Le blanc, comme le jaune, est signe de clarté, de lumière et de dévoilement ${ }^{14}$, tandis que le noir symbolise l'invisibilité, la méfiance face aux ennemis, le camouflage. Le bleu, « c'est passivité, sagesse, deuil, superstition, tout ça ». Quant à l'orange, il renvoie au langage didactique et sert à garnir le fond de nombreuses planches, comme à teindre l'habit de certains personnages. Une fois terminé le croquis en noir et blanc, Dialiba Konaté réfléchit à l'ambiance qu'il veut y faire sentir avant d'y mettre des couleurs.

Le choix pour les couleurs, dont il indique utiliser cinq nuances pour chaque couleur primaire et binaire, ajoute à la dimension mystique de l'œuvre, et c'est sur ce point qu'il insiste lors de notre deuxième entretien, précisant sa volonté de transmettre non seulement un savoir sur le passé aux jeunes générations en quête de racines, mais surtout des valeurs de respect et de vie qu'il sait lire dans un geste ou dans un objet posé là comme un signe. C'est cependant en toute modestie qu'il annonce son ignorance, face au savoir des initiés qu'il admire. En effet, si le dessin est pour lui une manière d'évoquer des symboles qui le frappent, il avoue aussi ne pas connaître l'ampleur de ce qu'à l'aide de ses crayons, il peut évoquer dans ses dessins. Renvoyant aux "paroles très anciennes " et citant Sory Camara dont il a également reçu des encouragements pour son œuvre, il raconte comment un vieux chasseur a réagi face au dessin qu'il lui présentait :

J'ai montré ce dessin à un vieux chasseur, les costumes des temps. Vous savez ce qu'il a dit? Vous savez quand eux ils lisent, les lignes, les traits, tout ça c'est sacré. Il a regardé les dessins longuement. Il a dit : à cette époque, chacun avait sa place; il y avait le respect. C'est comme ça qu'il a dit, pas autre chose. Il a regardé seulement le dessin, il a compris. Pas besoin de l'écriture. Il connaît le Lui il connaît mieux que ce que je fais. C'est des gens qui sont confidents aux choses, aux signes sacrés et tout, surtout l'homme, la constitution de l'homme, tout ce qui est sacré, comment c'est créé et tout. Il a regardé longuement les dessins et après il a dit: à cette époque là, chacun avait sa place (rire).

(Entretien avec Dialiba Konaté, 6 novembre 2009) 


\section{Ce que fait l'art de Dialiba Konaté}

21 agissant comme révélateur de valeurs et de secrets dont lui-même, n'étant pas à proprement parler ce qu'il appelle un «initié », n'aurait pas la maîtrise. Dialiba Konaté se défend de tout travail d'imagination: il ne fait que mettre sur papier cartonné le récit en couleurs d'aventures que l'on peut entendre raconter auprès de certaines personnes dont il recherche la présence, depuis ses années d'enfance passées chez son grand-père maternel.

L'intention du dessinateur, telle qu'on peut la déduire des entretiens menés avec lui, est seulement et en toute modestie de transmettre autrement un message qu'il a luimême reçu, comme pourrait le faire un griot (l'histoire des anciens, plus exactement des temps préislamiques: c'est pour cette raison qu'il s'est lancé dans l'empire du Ghana), mais aussi de transmettre des valeurs comme pourrait le faire un « initié » aux savoirs les plus ésotériques hérités du passé ou, plus simplement peut-être, un grandpère qui souhaiterait laisser un message de vie à ses enfants. La valeur communicative de son travail est première pour Dialiba Konaté, et il s'agit pour lui de dire, de transmettre et d'agir bien plus que de produire quelque chose d'esthétique ou d'artistique. Cependant, comme le fait bien remarquer Marie-Dominique Popelard, si le créateur ne se définit pas lui-même comme artiste, c'est dans la communication que la production devient œuvre d'art ${ }^{15}$.

Dialiba Konaté présentant ses derniers dessins à Étienne Féau (octobre 2009)

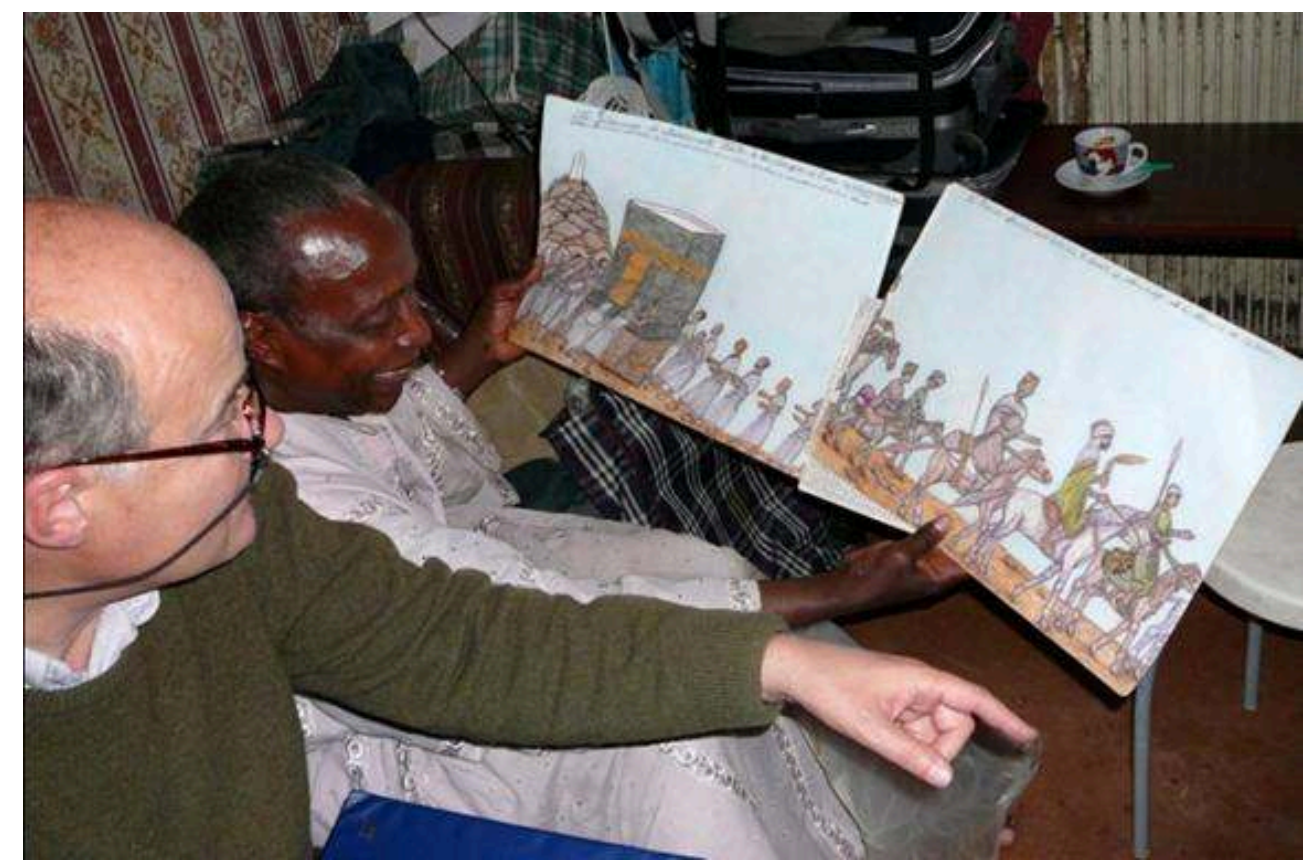

Les « valeurs africaines » auxquelles Dialiba Konaté se réfère sont pétries d'islam car il est lui-même musulman, fils de marabout, mais ce sont aussi les valeurs ancestrales de l'Afrique traditionnelle, telles que la représentent à ses yeux les «initiés » comme ce savant guérisseur rencontré autrefois dans le train de Dakar. Dans ses récits, la filiation entre empires de l'Afrique de l'Ouest et islam est posée comme une évidence, et ce de 
deux manières: d'une part, parce que les riches commerçants du Wagadu avaient éprouvé la nécessité de pouvoir compter leurs richesses et avaient ainsi envoyé leurs enfants chez les Arabes étudier les mathématiques. Ceux-ci auraient alors appris à connaître la religion du prophète en apprenant à compter, sans aucune violence. D'autre part, l'amitié du Prophète pour Bilal et les relations entre la région soudanaise et la péninsule arabe sont pour lui, comme pour beaucoup d'intellectuels africains dont il connaît les travaux, sources de motivation et de satisfaction. Cependant, les " vérités africaines " qu'il entend mettre en valeur à travers ses dessins sont ancrées dans des représentations préislamiques. Dialiba Konaté fréquente les chasseurs, les griots, les guérisseurs, les anciens, tous ces personnages auxquels il reconnaît des pouvoirs puissants, une familiarité avec la vie dans le moindre de ses mystères. Leurs discours sont au fondement de ce qu'il entend communiquer par son art, dans ce qu'il cherche à dire comme dans ce qu'il espère faire entendre sans qu'il soit nécessaire de le dire. Car tout ne saurait être dit si l'on est sage en Afrique (Diarra et Leguy, 2004), et cela même quand on parle avec des formes et des couleurs. Comme le rappelle Dialiba Konaté : "Les traditionalistes, surtout les griots, quand ils parlent sur l'homme, sur l'histoire, après ils disent : “j’ai tout dit, mais il en reste encore”. » (Entretien avec Dialiba Konaté, 6 novembre 2009)

Du point de vue de la réception, il est intéressant de relever le succès que l'album sur l'épopée de Soundiata a pu avoir auprès des professionnels du livre pour enfants, que ce soit les libraires spécialisés ou les animateurs de médiathèques (cf. les entretiens donnés en annexe du mémoire d'Anne Godin), alors qu'il est considéré comme un ouvrage difficile d'accès tant par la complexité de ses textes que par l'originalité des dessins. Il est devenu un des incontournables parmi les livres de jeunesse sur l'Afrique en France aujourd'hui. L'objectif de l'auteur cependant est de pouvoir toucher les jeunes Africains. Il est très déçu des difficultés rencontrées au Mali par exemple, où l'exposition sur Soundiata n'a finalement toujours pas été présentée, malgré différentes démarches. Dialiba Konaté raconte également qu'un de ses amis a vu dans une librairie l'album sur l'empire de Soundiata au Brésil, où il semble être plus facile à trouver qu'en Afrique de l'Ouest. Que les enfants du Mali ne puissent pas accéder aux récits colorés qu'il donne des épopées ancestrales peine profondément le dessinateur.

En donnant à voir par le dessin les récits légendaires des héros fondateurs des empires ouest-africains, Dialiba Konaté fait œuvre originale. S'il se présente comme un simple passeur de parole - comme se définissent généralement les acteurs de l'oralité dans ce contexte -, un homme qui a eu la chance de fréquenter des érudits locaux lors de sa jeunesse à Bandiagara et qui met ses connaissances au service des jeunes générations grâce à son don pour le dessin, il offre cependant, par l'abondance des tableaux exécutés et la profusion de détails qu'ils contiennent, une possibilité inédite que la parole puisse être transmise par d'autres voix que la seule transmission orale. Il refuse de nommer art ce qu'il considère comme un travail d'historien en recherche de vérité, mais c'est en véritable artiste qu'il œuvre pour que la parole continue d'être transmise, non seulement aux nouvelles générations, mais aussi à travers l'espace aux enfants du monde qui aiment qu'on leur lise et raconte les incroyables histoires vraies des héros légendaires de l'humanité. 


\section{BIBLIOGRAPHIE}

BA, Amadou Hampâté, 1994, Oui mon commandant. Mémoires II, Actes Sud (Babel).

CAMARA, Sory, 1982, Paroles très anciennes, ou le mythe de l'accomplissement de l'homme, Grenoble, La

Pensée sauvage.

CAMARA, Sory, 1992, Gens de la parole. Essai sur la condition et le rôle des griots dans la société malinké, Paris, Karthala.

Cissé, Youssouf Tata et КАміssоко, Wâ, 1988, La grande geste du Mali, Paris, Karthala-Arsan.

Cissé, Youssouf Tata et KАмISSOKо, Wâ, 1991, Soundjata, la gloire du Mali, Paris, Karthala-Arsan.

DERIVE, Jean (dir.), 2002, L'épopée. Unité et diversité d'un genre, Paris, Karthala.

DiABATÉ, Massa Makan, 1975, L'aigle et l'épervier ou la geste de Sunjata, Paris, Pierre-Jean Oswald.

DiARRA, Pierre et LEGUY, Cécile, 2004, Paroles imagées. Le proverbe au croisement des cultures, Rosnysous-Bois, Bréal (Langages \& Co).

FÉAU, Étienne, 2000, Soundiata Keïta : dessins de Konaté Dialiba, paroles de griots, Musée des arts d'Afrique et d'Océanie, Paris. [Catalogue de l'exposition de 2000-2001, Paris, Musée des arts d'Afrique et d'Océanie].

Godin, Anne, 2005, Les contes illustrés jeunesse d'Afrique noire dans le paysage éditorial et culturel français, Mémoire de l'Institut Universitaire de Technologie René Descartes, Université Paris 5. [consultable en ligne : http://www.ricochet-jeunes.org/public/fichiers/memoiregodin.pdf] INNES, Gordon, 1974, Sunjata: three mandinka versions, Londres, SOAS.

JANSEN, Jan, 1995, L'épopée de Sunjata, Leyde, CNWS.

JANSEN, Jan, 2001, Épopée, histoire, société. Le cas de Soundjata, Mali et Guinée, Paris, Karthala.

JoHnson, W. John, 1980, The Epic of Sonjara, Bloomington, Indiana University Press.

KonATÉ Dialiba (avec la collaboration de Martine Laffon), 2002, L'épopée de Soundiata Keïta, Paris, Seuil Jeunesse.

KonATÉ Dialiba (avec la collaboration de Martine Laffon), 2004, Le prince Maghan Diawara et le crocodile du lac Faguibine, Paris, Seuil Jeunesse.

KonaTÉ Dialiba (avec la collaboration de Martine Laffon), 2005, Le récit des origines, Paris, Seuil Jeunesse.

KonATÉ Dialiba (avec la collaboration de Martine Laffon), 2007, Les saisons oubliées : Une enfance africaine, Paris, Éditions du Panama.

KonATÉ Dialiba (avec la collaboration de Sylvie Baussier), 2010, La fabuleuse histoire de l'empire du Ghana, Paris, Seuil Jeunesse.

NAEF, Silvia, 2004, Y a-t-il une « question de l'image » en islam ?, Paris, Téraèdre.

NIANE, Djibril Tamsir, 1960, Soundjata, une épopée mandingue, Paris, Présence africaine.

POPELARD, Marie-Dominique, Ce que fait l'art, Paris, PUF.

ROBINSON, David, 1988, La guerre sainte d'Al-Hajj Umar, Paris, Karthala. 
TURNER, Victor W., 1972, « La classification des couleurs dans le rituel Ndembu : un problème de classification primitive », in Bradbury et al., Essais d'anthropologie religieuse, Paris, Gallimard, p. 67-107.

\section{NOTES}

1. Le griot qui fait cette remarque à Étienne Féau est Sourakata Koïté, originaire de Tambakounda, qui a enregistré plusieurs disques en France et aux Pays-Bas depuis les années 1970.

2. L'épopée de Soundiata a cependant fait l'objet en 1995 d'un film du réalisateur burkinabé Dani Kouyaté, griot qui envisage le cinéma comme un nouveau moyen donné aux griots pour faire entendre leurs paroles dans un monde où ils ne sont plus toujours écoutés : Keita! L'Héritage du griot, Afix Productions, Les Productions de la Lanterne, Sahelis (France/Burkina Faso). Dani Kouyaté s'est également intéressé aux récits soninké du Wagadu avec Sia, le rêve du python (2001), adaptation filmée de la pièce de théâtre du Mauritanien Moussa Diagana, La Légende du Wagadu vue par Sia Yatabéré (1988).

3. Sur la définition du genre en contexte africain, lire Jean Derive, « Le cas de l'épopée africaine » (2002).

4. Né en 1901, il avait servi de 1919 à 1925 comme secrétaire du premier gouverneur de Haute Volta, Édouard Hesling. Modibo Keïta fit appel à ses services de conseiller quand il prit le pouvoir après l'Indépendance.

5. Amadou Hampâté Bâ (1994) raconte sa rencontre avec Demba Sadio Diallo, «premier secrétaire indigène du gouverneur " (p. 118), qui organise une fête en son honneur le dimanche suivant sa prise de service au Bureau des enregistrements et des domaines à Ouagadougou et qu'il appelle ensuite «mon ami » (p.136: «Le Peul Demba Sadio Diallo, premier secrétaire du gouverneur, était devenu un de mes meilleurs amis. »)

6. Demba Sadio Diallo est également cité par David Robinson (1988) à qui il avait accordé un entretien en 1976. Selon Robinson, il était l'arrière-petit-fils de Juka Sambala Jallo (ou Diouka Sambala Diallo) de Médine (ville située dans la région de Kayes qui fut la première capitale du Haut-Sénégal-Niger), roi du Khasso qui avait permis au gouverneur Faidherbe de construire un fort pour lutter contre El Hadj Oumar Tall en 1855.

7. Étienne Féau, qui était alors conservateur au Musée des Beaux-Arts d'Angoulême, avait invité Dialiba Konaté à exposer l'histoire de Soundiata au Salon de la BD en janvier 1983. Malgré le peu d'enthousiasme manifesté à l'époque par les professionnels de la bande dessinée, le dessinateur ne s'est pas découragé et garde un bon souvenir de son passage à Angoulême.

8. Cf. catalogue de l'exposition.

9. L'exposition avait été montée avec la collaboration de l'Association MC2A (Migrations culturelles Aquitaine Afrique) dirigée par Guy Lenoir et fut présentée dans le courant de l'année 2001 à Bordeaux.

10. À vrai dire, Dialiba Konaté ne s'est jamais découragé face au travail titanesque dans lequel il s'est lancé dès ses premières années de présence en France, en suivant des cours de dessin et en accumulant les lectures érudites. Il signale lui-même le soutien reçu de la part des ambassadeurs du Sénégal et du Mali - ainsi que du Président Senghor dont il conserve précieusement la lettre d'encouragement - quand il les a informés de l'obtention de ses premières Unités de Valeur universitaires.

11. Kangaba est une petite ville située à quatre-vingt-quinze kilomètres au sud-ouest de Bamako, au bord du Niger, près de la frontière de la Guinée, dont les griots, surtout ceux du village voisin de Kéla, sont considérés comme « les détenteurs et les récitants officiels de la tradition relative à 
Soundjata » (Jansen, $2001:$ 11). Nombreux sont les griots qui viennent apprendre auprès des griots de Kangaba, ce qui leur assure un certain prestige.

12. Lors de l'entretien que j'ai avec lui le 26 octobre 2009, il me présente cependant un nouveau Coran illustré pour enfants, en me disant que « ça commence à se faire ».

13. Les principales raisons évoquées pour justifier cette interdiction sont émises en réaction à la condamnation de l'idolâtrie, les images étant considérées comme impures. Il n'est donc pas possible de prier en leur présence. Par ailleurs, les artistes produisant des images d'êtres humains ou d'animaux se rendraient coupables "d'immodestie » en voulant imiter Dieu, seul créateur, et risqueraient pour cela la condamnation dans l'au-delà. Les représentations ne comportant ni personnages ni animaux sont en revanche admises (Naef, 2004).

14. «La couleur blanche c'est la lumière, c'est un peu comme jaune, pour dévoiler des choses qui sont cachées, pour nous les Africains (rire). » (Entretien, 6 novembre 2009).

15. «À partir de la ressource (munis) qu'est l'œuvre, la communication (cum-munis) la transforme en œuvre d'art, elle la rend commune aux actants de la communication ainsi reliés. » (Popelard, 2002 : 104)

\section{RÉSUMÉS}

Après une exposition au Musée National des Arts d'Afrique et d'Océanie et la publication de grands albums illustrés dans des collections pour la jeunesse, Dialiba Konaté s'est imposé en proposant une œuvre particulièrement originale, utilisant le dessin pour transmettre les paroles des griots et donnant ainsi un support inédit aux légendes ancestrales de l'Afrique de l'Ouest. Nous nous interrogerons sur «ce que fait l'art» de Dialiba Konaté, à partir de ses sources d'inspiration, mais aussi de ses interrogations sur la représentation imagée et sur le choix des couleurs.

Following an exhibition at the Musée National des Arts d'Afrique et d'Océanie and the publication of illustrated albums for young people, Dialiba Konaté has become well known as a rather original artist who uses drawings to transmit words of the griot and thus provides significant support for the ancestral legends of West Africa. This article analyzes what Dialiba's "art actually does," based on his sources of inspiration, his questions about imaging, and on his choice of colors.

\section{INDEX}

Mots-clés : Konaté Dialiba (1942-), livres d'images, dessin, couleurs, littérature jeunesse

Keywords : West Africa, Drawings, Dolors, Konaté Dialiba (1942-), Pictures Book for Children, Graphic Arts, Anthropology, Oral Literature, Griots, Children's Literature

Index géographique : Afrique de l'Ouest

Thèmes : arts graphiques, littérature orale, anthropologie (Afrique) 